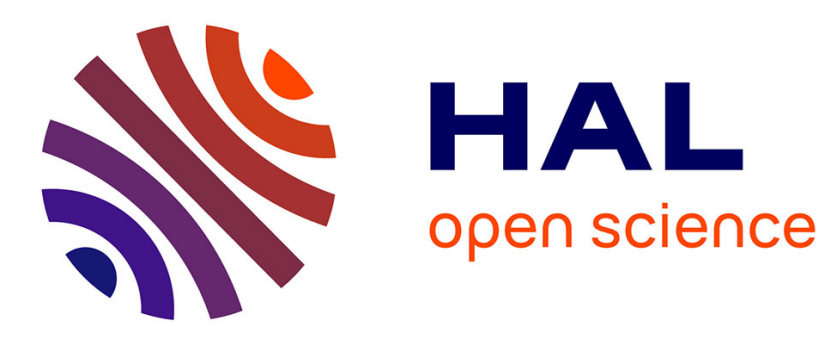

\title{
Extracting displacement and strain fields from checkerboard images with the Localized Spectrum
} Analysis

Michel Grediac, Benoît Blaysat, Frédéric Sur

\section{- To cite this version:}

Michel Grediac, Benoît Blaysat, Frédéric Sur. Extracting displacement and strain fields from checkerboard images with the Localized Spectrum Analysis. Experimental Mechanics, 2019, 59 (2), pp.207218. 10.1007/s11340-018-00439-2 . hal-01926054

\section{HAL Id: hal-01926054 \\ https://hal.science/hal-01926054}

Submitted on 16 Dec 2018

HAL is a multi-disciplinary open access archive for the deposit and dissemination of scientific research documents, whether they are published or not. The documents may come from teaching and research institutions in France or abroad, or from public or private research centers.
L'archive ouverte pluridisciplinaire HAL, est destinée au dépôt et à la diffusion de documents scientifiques de niveau recherche, publiés ou non, émanant des établissements d'enseignement et de recherche français ou étrangers, des laboratoires publics ou privés. 


\title{
Extracting displacement and strain fields from checkerboard images with the Localized Spectrum Analysis
}

\author{
Michel Grédiac ${ }^{1}$, Benoît Blaysat ${ }^{1}$, Frédéric Sur $^{2}$ \\ ${ }^{1}$ Université Clermont Auvergne, SIGMA, Institut Pascal, UMR CNRS 6602 \\ BP 10448, 63000 Clermont-Ferrand, France \\ ${ }^{2}$ Laboratoire Lorrain de Recherche en Informatique et ses Applications, UMR CNRS 7503 \\ Université de Lorraine, CNRS, INRIA projet Magrit, Campus Scientifique, BP 239, 54506 \\ Vandoeuvre-les-Nancy Cedex, France
}

\begin{abstract}
The performance of white-light full-field measurement methods strongly depends on the nature of the pattern used to mark the surface on which displacements and strains are measured. Finding optimized patterns is therefore a topical question. The aim of this study is to examine the case of the checkerboard pattern. It is first shown that this periodic pattern can be processed with a Fourier-based technique such as LSA. Experiments are then carried out to compare the noise level in displacement and strain maps obtained by processing classic 2D grid and checkerboard images. The conclusion is that the noise level observed in displacement and strain maps is significantly lower with a checkerboard than with a classic $2 \mathrm{D}$ grid. A notched specimen is finally tested to illustrate that very low strain levels can be measured with checkerboard patterns.
\end{abstract}

Keywords: checkerboard, digital image correlation, grid method, heteroscedastic noise, localized spectrum analysis, metrology, optimal pattern, pattern optimization, windowed Fourier transform

This is the author-manuscript version of

M. Grédiac, B. Blaysat, F. Sur. Extracting displacement and strain fields from checkerboard images with the Localized Spectrum Analysis. Experimental Mechanics, SEM \& Springer, 2019.

DOI: $10.1007 / \mathrm{s} 11340-018-00439-2$ 


\section{Introduction}

Because of their versatility and ease of use compared to interferometric techniques, full-field measurement systems based on white light illumination have rapidly spread in the experimental mechanics community. In particular, Digital Image Correlation (DIC), whose basic version only requires minimal surface preparation such as the spray-painting of random speckles onto the tested specimen, is now routinely employed in numerous cases for which displacement or strain fields bring a real added value to characterize the behavior of materials and structures. Another approach is to employ regular patterns instead of random ones, and to process such patterns by using a Fourier-based method instead of classic DIC. As discussed recently in [1], checkerboards constitute the optimal pattern in terms of sensor noise propagation if they are correctly sampled. This is due to the fact that propagation of the sensor noise of the camera to the final displacement and strain maps is minimized if the average image gradient is maximized, as predicted by the models available in the literature to describe this noise propagation $[2,3,4,5]$. Such a pattern is however not random but periodic. It is therefore not used in practice in DIC because the underlying algorithms may converge to a local minimum. DIC is nothing but a technique which minimizes iteratively the optical residual in the real domain. In a recent paper aimed at comparing some of the metrological parameters characterizing classic subset-based DIC used with speckles on the one hand, and a Fourier-based method named Localized Spectrum Analysis (LSA) used with 2D grids on the other hand [6], it has been shown that under mild assumptions, finding the displacement field that minimizes the optical residual is quasi-direct in the Fourier domain if the texture is a periodic pattern like a $2 \mathrm{D}$ grid. In this context, the objective of this study is to examine how to process another type of periodic pattern, namely the checkerboard, with a Fourier-based technique in order to extract displacement and strain fields. It is shown here that LSA can be used for this purpose.

The paper is organized as follows. The basics of LSA are first briefly recalled. It is then explained how this technique can be used to extracted displacement and strain fields from checkerboard images. The noise level in displacement and strain maps is then compared with the noise level in their counterparts obtained with classic $2 \mathrm{D}$ grids. It is shown that using checkerboards instead of $2 \mathrm{D}$ grids leads to lower noise levels in the measurement fields. Finally, the determination of the strain field near a crack tip of a notched specimen illustrates the efficiency of this technique in a real case.

\section{A brief reminder on the Localized Spectrum Analysis}

We present here briefly the fundamentals of LSA. LSA consists first in calculating the windowed Fourier transform (WFT) of the image of the periodic pattern for a given frequency, which is the nominal frequency of the periodic pattern. This frequency is denoted by $f=\frac{1}{p}$, where $p$ is the pitch of the pattern. For a periodic pattern aligned with the $x$ and $y$ axes, the WFT can be written as follows

$$
\left.\widehat{s_{g}}(x, y, \theta)=\int_{-\infty}^{+\infty} \int_{-\infty}^{+\infty} s(\eta, \xi) g(x-\eta, y-\xi) e^{-2 i \pi f(x \cos \theta+y \sin \theta}\right) d \eta d \xi,
$$

where $s$ is the gray level distribution of the image, $g$ is a window centered at the pixel of coordinates $(x, y)$ where $\widehat{s_{g}}(x, y, \theta)$ is calculated. The Gaussian window constitues the best 
tradeoff between various constraints [7]. The function defining this Gaussian window is given by the following equation:

$$
g(x, y)=\frac{1}{2 \pi \sigma^{2}} e^{\left(-\frac{x^{2}+y^{2}}{2 \sigma^{2}}\right)},
$$

where $\sigma$ is the standard deviation of the Gaussian. $\sigma$ can be considered as a handy parameter which governs the apparent width of the window used in LSA, this quantity being equal to $6 \sigma$ according to the so-called " $3-\sigma$ rule" [8]. $\widehat{s_{g}}$ is calculated for two angles, namely $\theta=0$ and $\frac{\pi}{2}$, to get the information along the $x$ and $y$ directions, respectively. Each of these two calculations provides a distribution of complex numbers defined pixelwise. The displacements along the $x$ - and $y$-directions, denoted respectively by $u_{x}$ and $u_{y}$, are proportional to the phase change between current (or deformed) and reference images [9, 10]. Thus

$$
\underline{u}(\underline{x})=-\frac{p}{2 \pi}\left(\underline{\Phi}^{c u r}(\underline{x}+\underline{u}(\underline{x}))-\underline{\Phi}^{r e f}(\underline{x})\right),
$$

where $\underline{u}$ denotes the displacement vector of components $u_{x}$ and $u_{y}$ in the $(x, y)$ basis. $\underline{\Phi}^{\text {cur }}$ and $\Phi^{r e f}$ denote the current and reference phase vectors of components $\Phi_{x}^{r e f}, \Phi_{y}^{r e f}$ and $\Phi_{x}^{\text {cur }}$, $\Phi_{y}^{\text {cur }}$ in the $(x, y)$ basis, respectively. $\underline{u}$ is involved in both parts of the equality. It is therefore retrieved by using the fixed-point algorithm, which rapidly converges here. The main reason is that the convergence of the algorithm is fast when the first derivatives of $u$ are small, which is the case here. In practice, only one iteration is required to reach convergence in the case of small strains, as discussed in [11]. It is generally admitted that the phase distributions $\Phi_{x}$ and $\Phi_{y}$ of the reference and current images of the regular pattern are equal to the argument of $\widehat{s_{g}}(x, y, 0)$ and $\widehat{s_{g}}\left(x, y, \frac{\pi}{2}\right)$, up to a constant value. This constant value is the same for all these phase distributions. Thus it disappears when calculating the phase change to obtain the displacement with Equation 3. The measured phases are in fact equal to their true counterparts convolved by $g[11]$ but this effect is not considered here for the sake of simplicity. In addition, this does not change the conclusion of this study.

Finally, it is shown in [12] that the standard deviation of the noise in the phase maps can be predicted by using the following equation

$$
\sigma_{\Phi}=\frac{\sigma_{\text {image }}}{2 \sqrt{2 \pi} \sigma K} .
$$

This noise is assumed to be homoscedastic in this calculation. In this equation, $K$ is the modulus of the WFT and $\sigma_{\text {image }}$ the standard deviation of the noise impairing the images. $K$ is equal to $[12]$

$$
K=\left|d_{1}\right| \frac{\gamma A}{2},
$$

where $A$ is the amplitude of the periodic signal, $\gamma$ the contrast and $\left|d_{1}\right|$ the coefficient of the first harmonic in the development in Fourier series of the periodic signal.

LSA was used so far to retrieve displacement and strain fields from 2D grid images, so let us now examine how to adapt this technique to process checkerboard images. 


\section{Employing LSA to retrieve in-plane displacements from checker- board images}

The objective here is to examine how LSA can be used to process checkerboard images in order to retrieve displacement and strain fields. Two procedures are proposed and studied in this section.

\subsection{Procedure 1}

The first procedure is based on the following observation: when considering a checkerboard along the \pm 45 degree directions, two sets of perpendicular lines are clearly visible, forming each a $1 \mathrm{D}$ grid along one of the bisectors of the $x$ and $y$ directions. The corresponding directions are denoted $x^{\prime}$ and $y^{\prime}$. As an illustration, Figure 1-a and -b show a schematic view of these two 1D grids. The border of the "lines" forming these two 1D grids is not straight since the lines of these grids are made of diamonds placed side by side (see in Figure 1-c a closeup view of Region A defined in Figure 1-b). These lines interlock, and the pitch of these $1 \mathrm{D}$ grids

is equal to $p \frac{\sqrt{2}}{2}$, where $p$ is the pitch of the checkerboard along the $x$ and $y$ axes. Though these lines do not have straight borders, they form along each of the $x^{\prime}$ and $y^{\prime}$-directions a periodic pattern similar to a $1 \mathrm{D}$ grid. As such, these patterns can be processed with LSA applied along these two directions, with a frequency equal to $\frac{\sqrt{2}}{p}$. The displacement along the $x^{\prime}$ and $y^{\prime}$ directions is then derived from the phases, and the displacement along the $x$ and $y$ directions is deduced by applying a mere change of basis.

\subsection{Procedure 2}

The second procedure consists of preprocessing the checkerboard to extract two perpendicular $1 \mathrm{D}$ grids along the $x$ and $y$ directions. LSA is then applied in order to extract the phases along these two directions, and finally to deduce the displacement and strain fields directly in the $x-y$ basis from these phase fields.

Indeed, a 1D grid made of vertical lines can be created from a checkerboard by flipping the sign of the gray level of each pixel of the checkerboard with respect to the mean value of the gray level distribution. To obtain a vertical grid, this sign flip is applied one line out of every two. This procedure is illustrated in Figure 2. The set of lines defining Domain $\mathcal{D}_{1}$ remains unchanged, while the pixels of the lines defining Domain $\mathcal{D}_{2}$ are affected by the sign flip. A similar procedure can be applied along the columns instead of the lines to obtain a horizontal grid. As a result, two perpendicular 1D grids can be deduced from any checkerboard. Each of these two "virtual" 1D grids can then be processed by using LSA to extract the phase (and thus the displacement) along the $x$ and $y$ directions. The sign flip applied here does not impact the phase change between current and reference images, and the information concerning the displacement is therefore unaffected. On the contrary, the sign flip increases the norm of the WFT since it is applied to a $1 \mathrm{D}$ grid. Indeed such a pattern is closer in shape to a pure sine than a checkerboard. This leads the value of $\left|d_{1}\right|$ in Equation 5 to be higher for a $1 \mathrm{D}$ grid, and so the modulus $K$. A higher modulus leads to a lower noise, as can be checked in Equation 4.

Figure 3 shows an example of a real checkerboard transformed in two 1D grids. These two grids are visually very close to ideal $1 \mathrm{D}$ grids, which is very favorable for the Fourier 


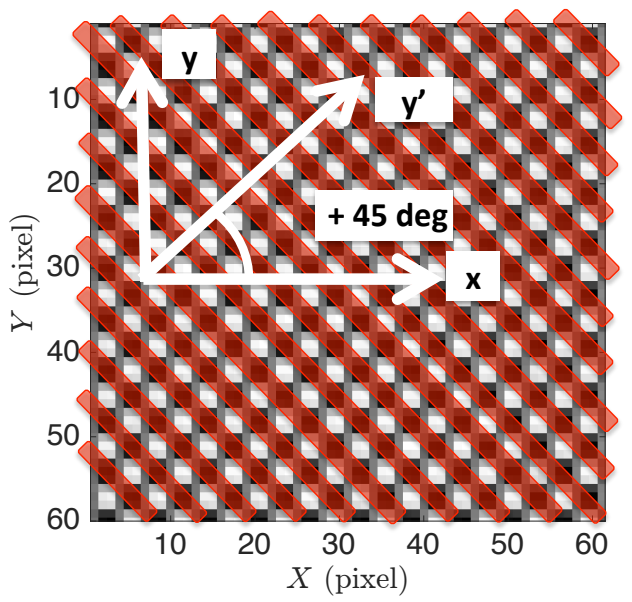

a-

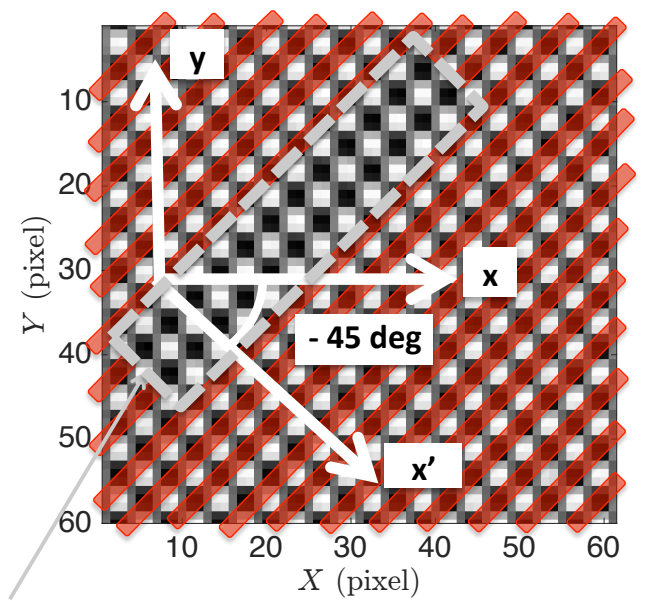

b-

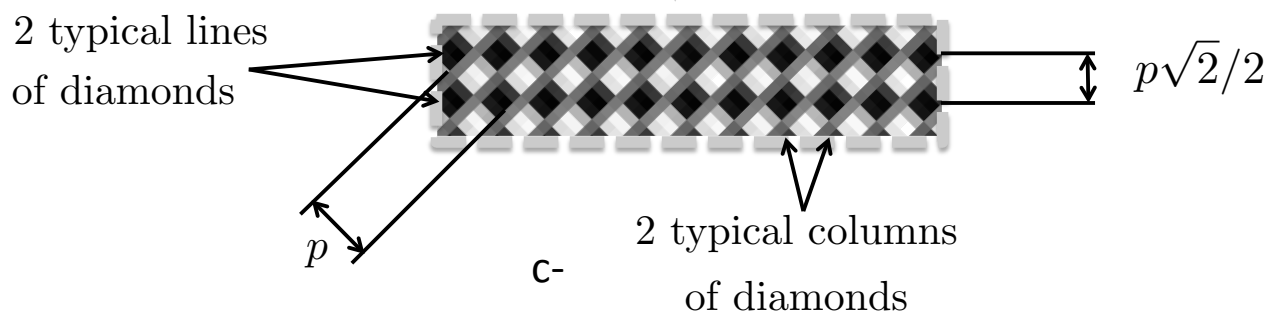

Figure 1: 1D grids observed along the bisectors of a checkerboard. a- along the +45 deg bisector. b- along the -45 deg bisector. c- closeup view of the diamond-like pattern observed in Region A along the -45 direction. Lines and columns of diamonds are clearly visible.

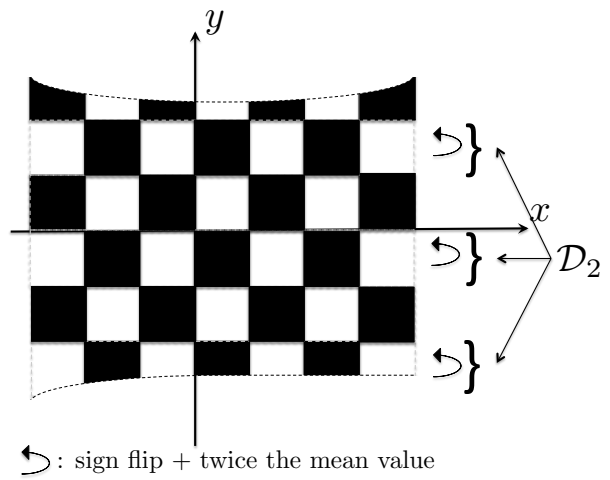

a-

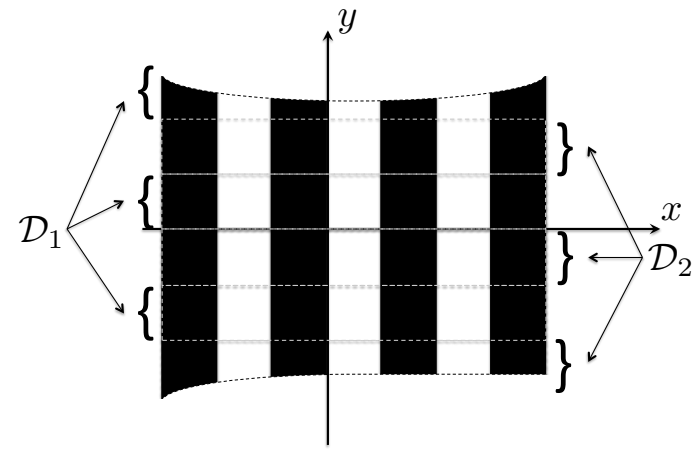

b-

Figure 2: From checkerboard to grid. a- Checkerboard. b- Vertical grid obtained by flipping the sign over $\mathcal{D}_{2}$ and adding twice the mean gray value. A similar procedure can be used to obtain an horizontal grid. 
analysis performed with LSA, even more favorable than when $2 \mathrm{D}$ grids are processed. The only apparent difference is the fact that the lines of these two 1D grids are not perfectly continuous, but serrated. This is clearly visible by plotting the gray level distribution along a dark and a bright column of pixels (columns 21 and 24 in Figure 3-b, respectively). This phenomenon comes from the pixels being at the border between black and white boxes in checkerboard images.

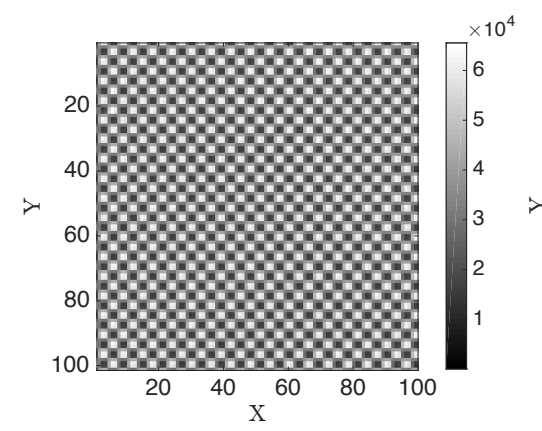

a-

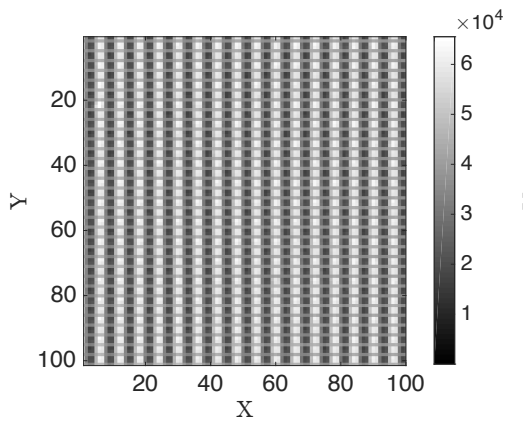

b-

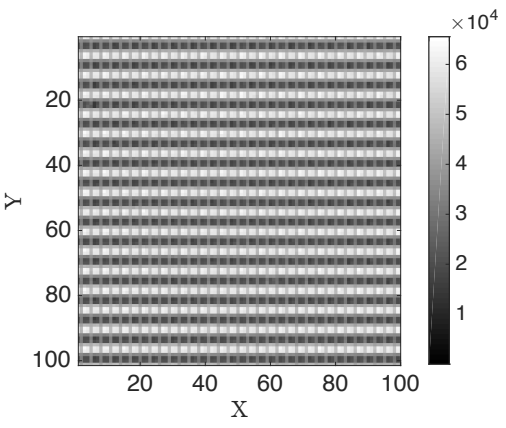

c-

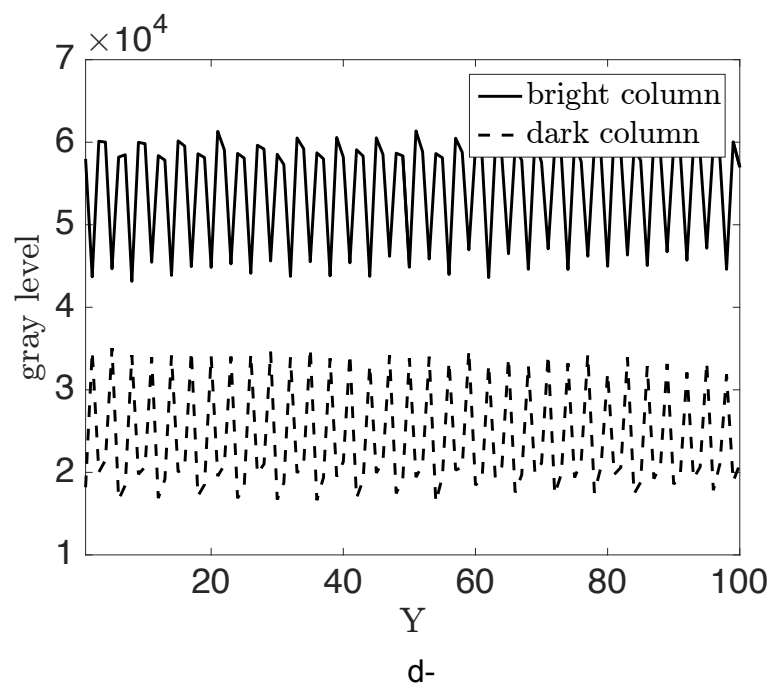

Figure 3: a- Closeup view of a real checkerboard image. b- and c- Closeup view of the 1D grids deduced from the checkerboard shown in a-. d- Vertical cross-section of the gray-level distribution along two columns of pixels in subfigure b- Bright column $x=21$ and dark column $x=24$. Dimensions in pixels.

\subsection{Comments on Procedures 1 and 2}

With Procedure 1, the border of the lines forming the inclined 1D grids is not straight, while the 1D grids deduced from the checkerboard with Procedure 2 are very close to ideal 1D grids. The modulus of the WFT, which reflects the "closeness" of a given periodic pattern to a pure sine profile and governs sensor noise propagation (see Equation 4), is therefore expected to be lower for Procedure 1 than for Procedure 2. The noise in final phase, displacement and 
thus strain fields are inversely proportional to this modulus. This potentially leads the fields obtained with Procedure 1 to be noisier than those obtained with Procedure 2. However, compared to Procedure 1, the pitch of two 1D grids deduced from the checkerboard is $\sqrt{2}$ times greater for the $1 \mathrm{D}$ grids deduced from Procedure 2 than for the \pm 45 -degree $1 \mathrm{D}$ grids of diamond-shaped patterns directly processed by Procedure 1. This feature negatively impacts the spatial resolution of Procedure 2 compared to Procedure 1. However the global metrological performance of a full-field measurement technique can advantageously be considered as a tradeoff between measurement resolution and spatial resolution for a given bias [11]. It is therefore difficult to guess which of the two techniques is the most efficient, and experiments should therefore be carried out to investigate this point specifically. This is the aim of the experiment described in the following section.

\subsection{Procedure 1 or Procedure 2, which is the best?}

Experiments were carried out to see which of the two procedures is the best in terms of noise affecting the displacement and strain maps. A checkerboard was deposited on a specimen using the technique described in [13]. The specimen was placed in a rigid metallic frame to measure noise obtained in DIC. This frame is equipped with a specific device, with which a horizontal translation can be applied to the specimen. This frame was placed on a marble slab used for metrology purposes. A Sensicam CCD camera was stiffly attached on the same frame to limit the relative displacement between specimen and camera. This is a 12-bit camera, but it provides TIF images encoded on 16 bits. The distance between camera and specimen was such that the pitch of the checkerboard, equal here to 200 microns (each black or white square is 100 microns in size), was equal to 6 pixels in the images. It means that all the features of these patterns, namely the black and the white squares forming the checkerboard, are on average 3 pixels in size in these images. This value is recommended in [23] to sample speckle dots in an optimal way with respect to aliasing in the case of DIC. Concerning checkerboards processed by LSA, the objective is to sample the quasi-periodic signal in such a way that it is as close as possible to a sine profile, and that it exhibits the maximum amplitude to have the highest contrast in the images. The first constraint means that choosing too many pixels should be avoided, the sampled profile tending to a rectangular profile as the number of pixels increases since this is the actual one. In addition, increasing the number of pixels per period reduces the size of the field of view in the same proportion. The second constraint means that too small a number should also be avoided, the PSF combined with the averaging effect of the gray levels at the scale of the pixels automatically reducing the amplitude of the signal. 3 pixels per square for the checkerboard seems a good choice in this case too since we are sure to have always at least one pixel corresponding to the brightest zone (the darkest zone, resp.) of the white squares (black squares, resp.). A smaller number could perhaps also be considered, but this option has not been investigated here because Procedure 2 requires that the squares of the checkerboard are sampled with a number of pixels close to an integer.

The specimen was illuminated with two LED light sources placed symmetrically, near the camera and along the two sides of the specimen. The specimen was fixed in such a way that the lines and columns of pixels were aligned with the rows of pixels of the camera. This makes easier the application of the preprocessing step described above for Procedure 2. Figure 4 shows the experimental setup.

200 pictures were taken in the reference configuration and 200 others after applying a 


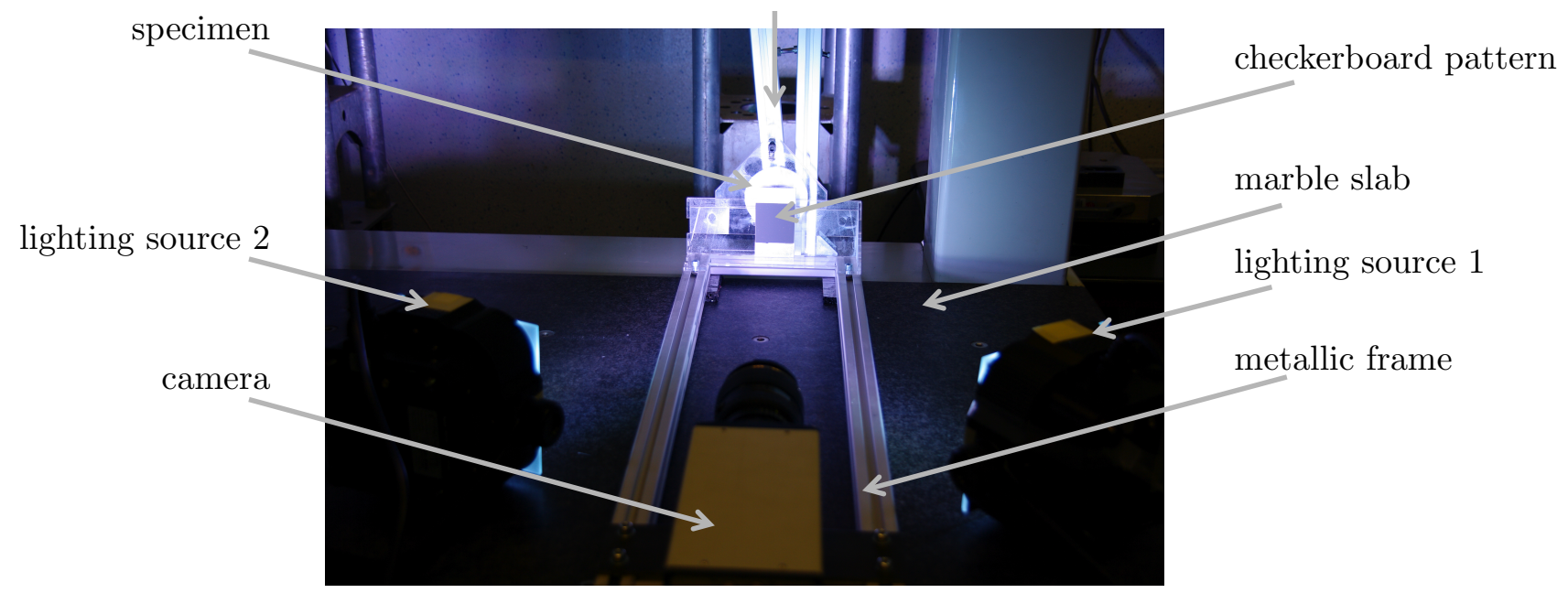

Figure 4: Setup used for the translation test

slight horizontal translation to the specimen. This stack of images enables us to calculate, for each of the two procedures described above, 200 displacement and strain maps. These maps can then be used for comparison purposes between both procedures. Figure 5 shows the histograms of the standard deviation calculated pixelwise from the stack of images, and by applying in turn Procedures 1 and 2 to process them. The equivalent standard deviation for the displacement, defined by

$$
\sigma_{\text {equ }}^{u}=\sqrt{\frac{1}{N} \sum_{k=1}^{N} s t d^{2}\left(u_{j}\left(x_{k}, y_{k}\right)\right)} \quad j=x \text { or } j=y
$$

is calculated and displayed in each case. This quantity reflects the average noise level in displacement maps. The same quantity is introduced for the strain components. It must be emphasized that performing reliable experiments is tricky, any tiny movement between camera and specimen influencing the estimation of the standard deviation of the displacement along time (strains are not concerned by such rigid-motion like movements). Though all the components of the measuring device were stiffly attached to the metallic frame, a tiny rigid-body like micro-movement was detected in the displacement maps. Consequently, the average displacement was subtracted from each displacement map to get rid of this parasitic movement.

The main remark, which can be drawn from the histograms shown in Figure 5, is that both procedures give nearly the same results. Since Procedure 1 is nearly two times faster because no pre-processing step is needed, it should be used instead of Procedure 2 when processing checkerboard images. It can also be seen that the histograms for the displacement feature some irregularities. This is likely a consequence of aliasing in the images. This phenomenon manifests itself by slight low-frequency parasitic fringes in the maps [14], but the location of the fringes slightly changes from one image to another in the stack, thus inducing temporal fluctuations of the displacement, which are not only due to the camera sensor noise. This is confirmed by the fact that the histograms for the strains shown in Figure 6 are smoother 

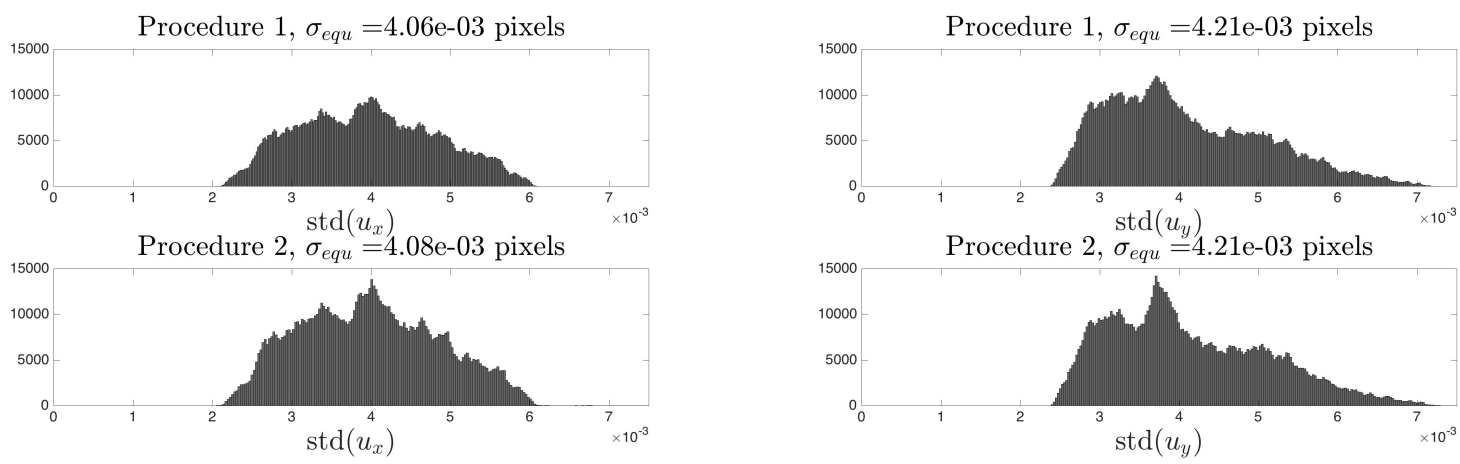

Figure 5: Histograms for the standard deviation of the $u_{x}$ and $u_{y}$ distributions, calculated pixelwise as a function of time. Dimension: pixel, thus the quantities reported along the horizontal axis shall be multiplied by $200 / 6$ to have results in microns

than those for the displacements shown in Figure 5. Indeed aliasing causes very low-frequency spatial fluctuations in the displacement maps, so their influence when spatially differentiating to retrieve the strains is negligible. Removing the consequence of aliasing in the displacement and strain maps is obtained by rotating the checkerboard, as in [14]. In this case, the flipping procedure used in Procedure 2 becomes much more complex compared to the one used in the present experiment where the checkerboard is aligned with the camera sensor. It would also certainly be less accurate because interpolations should be made, and even slower. This is an equally important reason to choose Procedure 1 instead of Procedure 2. Finally, it is worth noting that the order of magnitude of the standard deviation measured here for the displacement is $4 \mathrm{E}-03$ pixel, which is equal to $200 / 6 \times 4 \mathrm{E}-03=0.133$ micron. This order of magnitude underlines the fact that reliably estimating such a noise level needs, among others, that every component of the device is stiffly attached. The conclusion of this first experiment is that only Procedure 1 will be used hereafter for comparison purpose with classic $2 \mathrm{D}$ grids but aliasing in the images should be carefully controlled.

\section{Comparing sensor noise propagations obtained for checker- boards and classic $2 \mathrm{D}$ grids}

The aim here is to assess experimentally the improvement brought about by using checkerboards instead of classic $2 \mathrm{D}$ grids used in many examples of in-plane displacement and strain measurements $[15,16,17,18,19,20,21]$ for instance. This comparison is performed here in terms of sensor noise propagation for a given size of window $g$ defined by Equation 2 above. This noise was estimated in each case from displacement and strain maps deduced from a stack of 200 images taken during a translation test [22], as in the preceding experiment.

\subsection{Experimental conditions}

Two different specimens were prepared for this experiment, the first one with a checkerboard and the second one with a $2 \mathrm{D}$ grid. Both types of regular patterns were deposited with the 

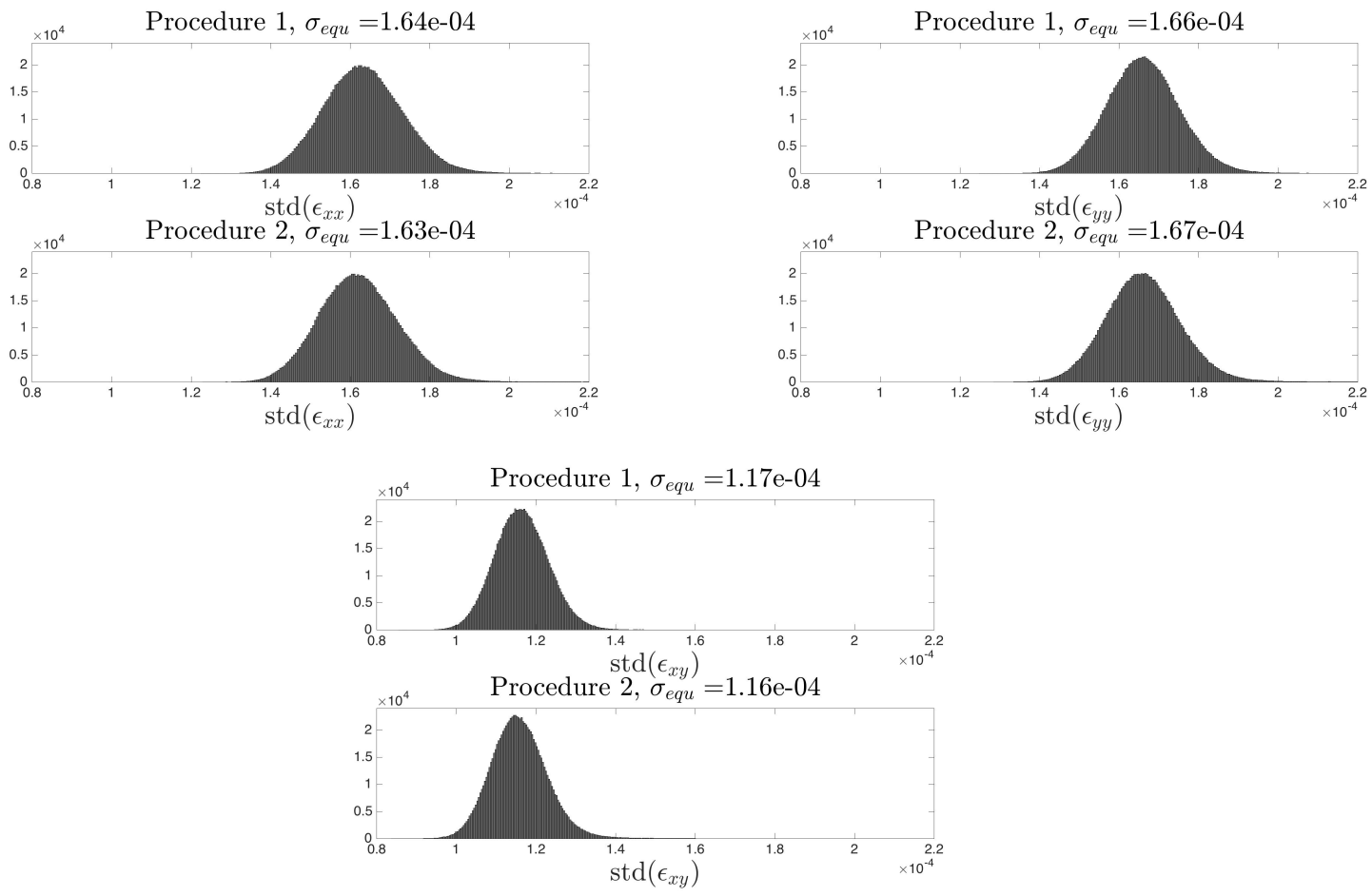

Figure 6: Histograms for the $\varepsilon_{x x}, \varepsilon_{y y}$ and $\varepsilon_{x y}$ distributions, calculated pixelwise as a function of time

procedure described in [13]. They were slightly inclined to avoid aliasing in the corresponding images, as justified in [14] and illustrated in Figure 7. As in the preceding case, the distance between camera and specimens was adjusted in such a way that 6 pixels were used to sample one grid and checkerboard period. The two specimens were fixed in turn in a grip and a translation was applied to each of them according to the procedure described in Section 3.4 above. The light intensity was adjusted in such a way that the highest dynamic range of the camera sensor was used, but without saturating any pixel. Interestingly, the optimal lighting conditions are not the same for the two types of specimens. Indeed the bright spots in a 2D grid image are darker than their counterparts in a checkerboard image if exactly the same lighting conditions and camera settings (aperture, shutter time) are used in both cases, although the size of these spots is exactly the same. This is probably a consequence of the point spread function (PSF) of the lens of the camera. Indeed, white boxes are completely surrounded by black lines in 2D grid images, which is not the case for the checkerboard. The aperture of the lens was therefore changed and the distance between specimen and lighting sources was adjusted from one case to each other, in order to have a gray level distribution, which covers the widest range without reaching saturation at any pixel.

The gray level distributions for these different specimens and lighting conditions for each of these specimens are shown in Figure 8. It can be seen that these distributions are completely different, but they span the widest possible dynamic range of the sensor since the right-hand tail is close to $2^{16}-1$. 


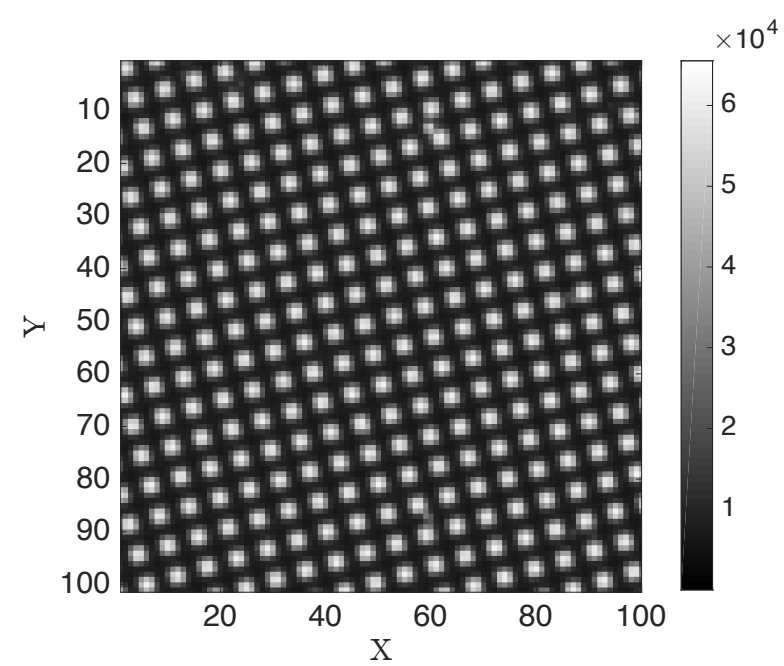

a- Classic 2D grid

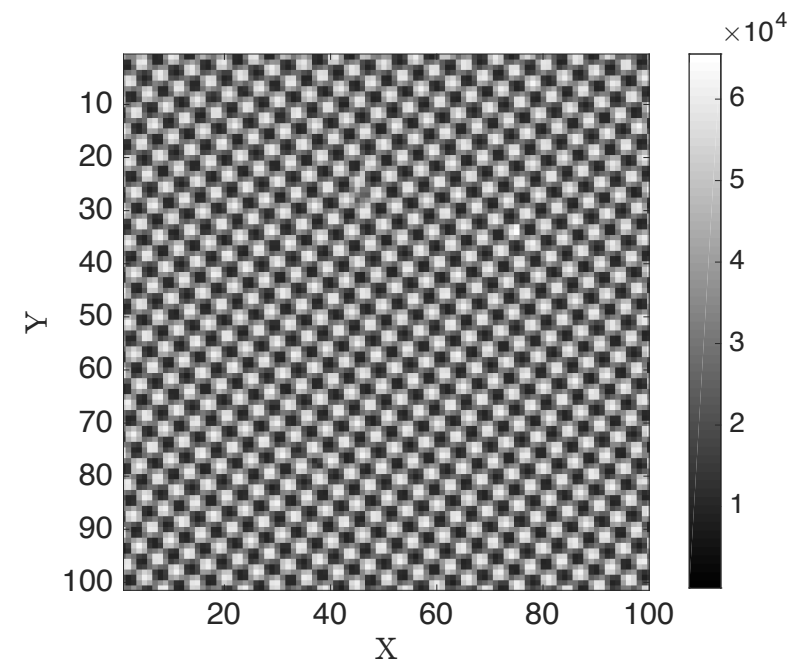

b- Checkerboard

Figure 7: Closeup views of the checkerboard and 2D grid patterns compared in this experiment. a-: 2D grid. b- Checkerboard. Dimensions in pixels.

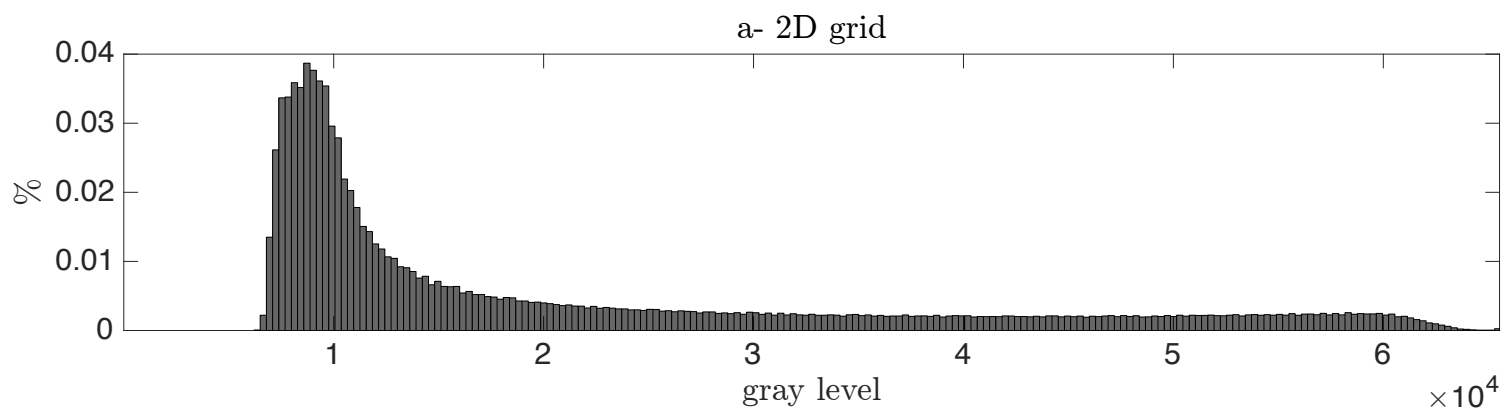

b- Checkerboard

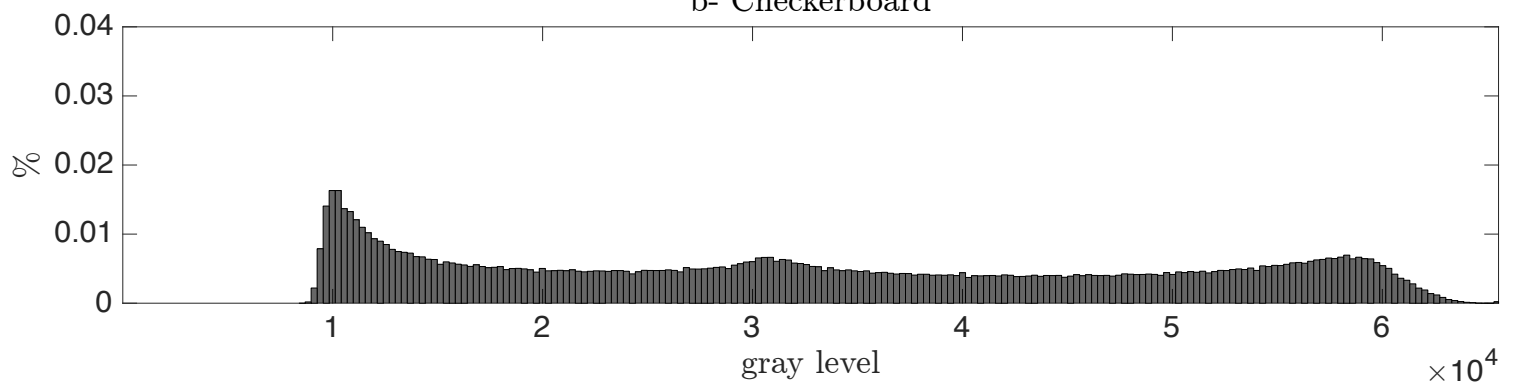

Figure 8: Histogram of the gray level distribution for 2D grid and the checkerboard. 
This difference in gray level distribution that is observed in these histograms also means that on average, the noise level is not the same in the images of these two types of patterns because camera sensor noise is heteroscedastic [24, 25, 26, 27], so the higher the brightness in the patterns, the higher the noise impairing the images. As shown in Equation 4, noise in the displacement and strain maps obtained with LSA is inversely proportional to the modulus of the Windowed Fourier Transform, but it is not easy to guess a priori if this modulus is greater for a checkerboard or for a $2 \mathrm{D}$ grid. In addition, the noise level in the maps is proportional to the noise in the image, and the latter is greater in checkerboard images than in 2D grid images. The combination of these two effects (amplitude of the WFT in the one hand, noise in the images in the other hand) should be considered when comparing these two types of patterns. Experiments are therefore necessary in order to more completely study the combined effects of these two phenomena.

\subsubsection{Displacement resolution}

Figure 9 shows the histograms of the empirical standard deviation deduced pixelwise from the stack of $200 u_{x}$ and $u_{y}$ maps. The equivalent standard deviation for the noise calculated from these standard deviation distributions is reported in each subfigure.
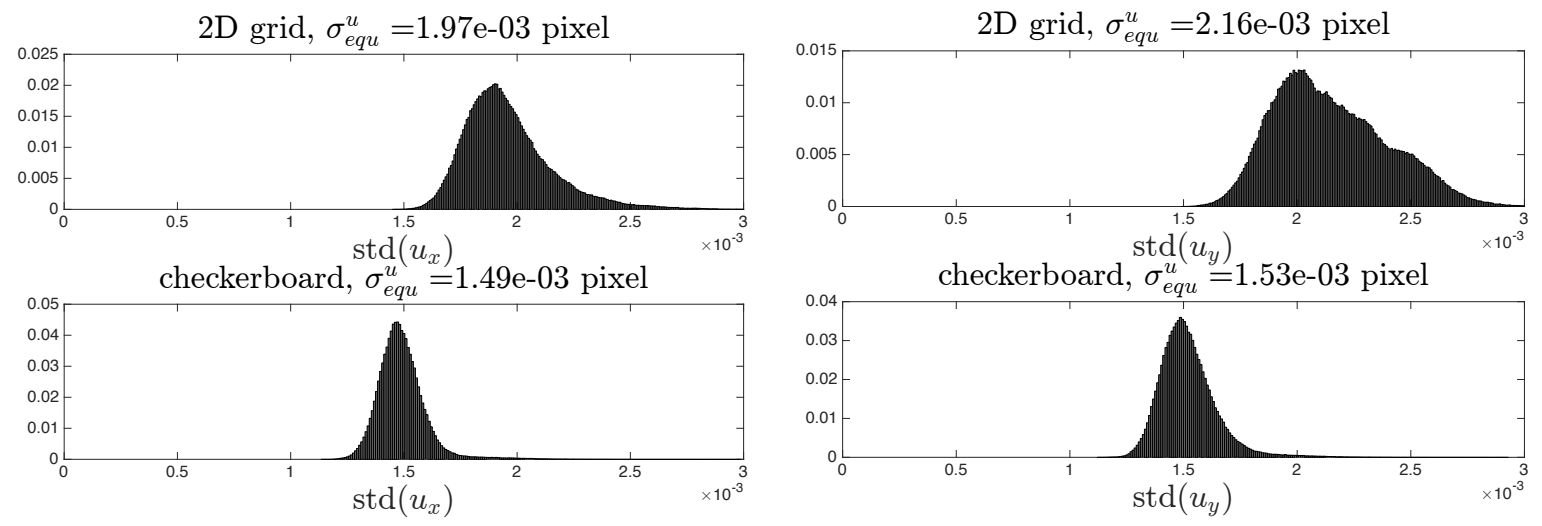

a- $u_{x}$

b- $u_{y}$

Figure 9: Normalized histogram of the noise in displacement maps obtained with the 2D grid and the checkerboard

The main conclusion is that the theoretical expectations discussed above are experimentally verified: the noise level in displacement maps obtained with the $2 \mathrm{D}$ grid is greater than the noise level in displacement maps obtained with checkerboard. The relative difference is equal to $32 \%$ for the $x$-direction and $41 \%$ for the $y$-direction. The difference in amplitude between the two directions is probably due to the effect of some micro-movements, which are less efficiently eliminated along the vertical direction.

\subsubsection{Strain resolution}

Strain components are deduced by direct differentiation of the displacement fields, which is possible here since the displacements are obtained pixelwise. The histograms for the noise in 
the strain maps are shown in Figure 10.
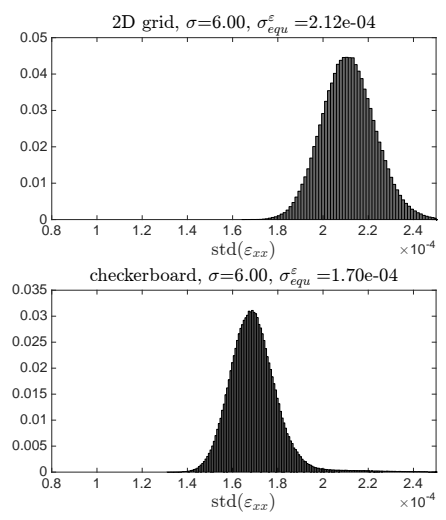

a- $\varepsilon_{x x}$
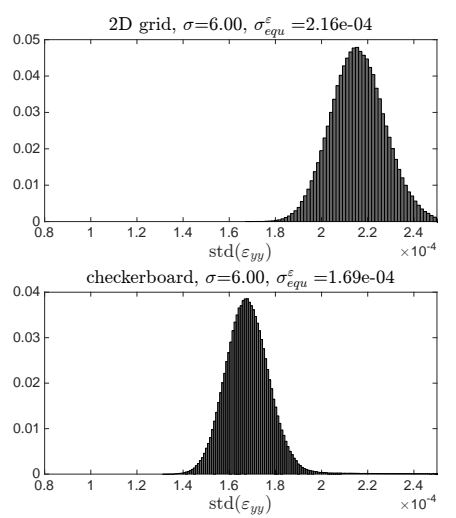

$\mathrm{b}-\varepsilon_{y y}$
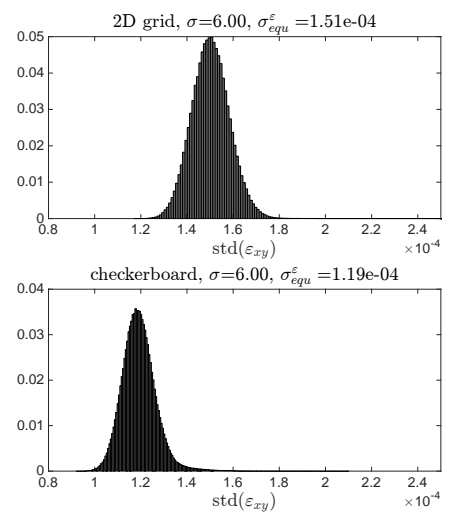

c- $\varepsilon_{x y}$

Figure 10: Normalized histogram of the noise in strain maps obtained with the 2D grid and the checkerboard

The ranking between the two techniques remains obviously unchanged. As expected, the equivalent standard deviation for the $x y$ strain component is about $\sqrt{2}$ lower than the equivalent standard deviations for the $x x$ and $y y$ components. As for the displacement, the noise affecting the strain maps is lower with a checkerboard than with a $2 \mathrm{D}$ grid.

\section{$5 \quad$ Strain field near a notch}

Previous experiments were mere translations applied on 2D grids and checkerboard, thus without any actual gradient in the strain maps to be measured. Here we propose to show that checkerboards and LSA can be used in real situations of material testing, by considering here as an example a tensile test carried out on a notched rectangular specimen. The objective is to measure high strain gradients at the very beginning of the test, thus in the case of low strain level. The information only barely emerges from the noise floor, so retrieving the actual details in the strain distribution is challenging. In this case, it is tempting to enlarge the size of the window used in the WFT to average out the noise, but this also induces a systematic error, which is all the higher as the strain gradient is high. The specimen tested here is rectangular (dimensions: $200 \mathrm{~mm} \times 40 \mathrm{~mm} \times 2 \mathrm{~mm}$ ). A notch (dimensions: $20 \mathrm{~mm} \times 1 \mathrm{~mm}$ ) was machined before the test at mid-height with a saw, perpendicularly to the loading direction. A schematic of the tested specimen and a closeup view of the surface under investigation are shown in Figure 11.

The checkerboard pattern was deposited using the procedure described in [13]. It was inclined by about 10 degrees with respect to the pixel grid of the sensor to avoid aliasing, as justified in [14] in the similar case of $2 \mathrm{D}$ grids. Localized defects of the periodic pattern are deliberately kept in this example so that the reader can see their impact on the final strain maps shown in the next figure. We employed here a camera with a sensor greater in size compared to the one used in the preceding example (PCO 2000 with a 4Mpixel CCD sensor). The number of pixels per period used to sample the signal was therefore higher than 

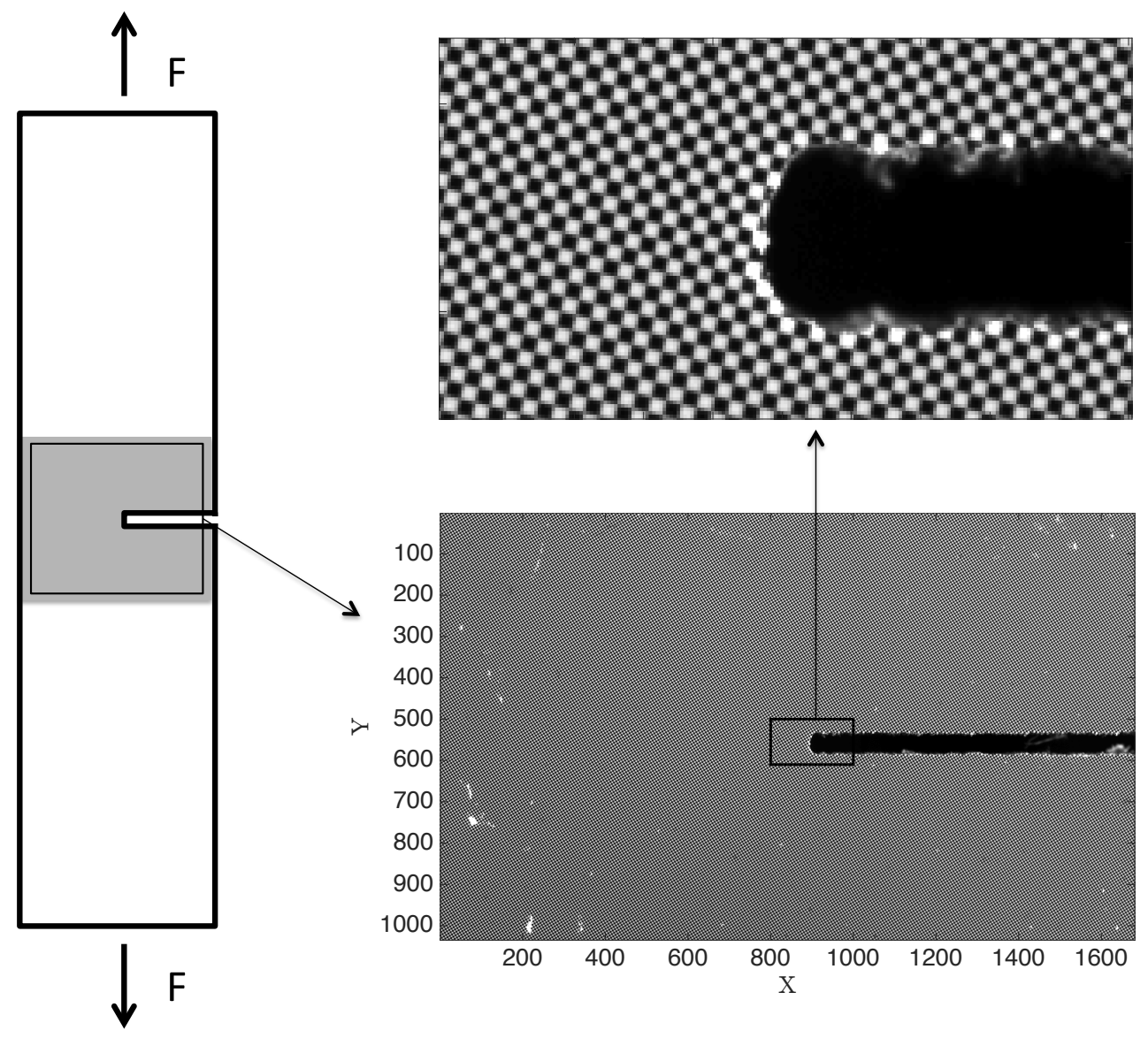

Figure 11: Notched specimen. Schematic, front view around the notch and close-up view of the zone near of the notch. The checkerboard is inclined to avoid aliasing in the images, as justified in [14] for classic 2D grids. Dimensions in pixels. The size of each black or white square of the pattern is $0.1 \mathrm{~mm}$

in the preceding example, namely 9 pixels per period along the natural axes of symmetry of the checkerboard instead of 6 in the preceding examples. Hence lower noise in the maps was obtained since the integral involved in the WFT is discretized over a greater number of points. The standard deviation of the Gaussian window used to process the images is equal to 7 pixels, which is slightly greater that the minimum value after [7] (equal to $9 \times \frac{\sqrt{2}}{2} \simeq 6.36$ pixels). To reduce the noise level in the final strain maps, the initial phase distribution $\Phi_{\text {ref }}$ involved in Equation 3 was obtained by averaging the phase distributions found with 100 successive frames. This is a simple way to systematically reduce the noise floor in strain maps. A slight movement was observed while taking these 100 images. It was removed in the corresponding phase distributions by subtracting the mean phase value. It is worth remembering that averaging 100 images and extracting then the corresponding phases is probably the most intuitive procedure, but the micro-movements occurring while taking the 100 images cause 
the resulting average image to a be a biased estimator of the noiseless image, as discussed in $[28]$.

The notched specimen was fixed in the grips of a Zwick-Roell tensile machine and subjected to a tensile test along the $y$-direction. The cross-head speed was $0.005 \mathrm{~mm} / \mathrm{s}$. Images were regularly taken during the test with a shutter time equal to $6 \mathrm{~ms}$ to avoid any blur in the checkerboard images. Typical strain maps are shown in Figure 12. As justified above, they are obtained at the very beginning of the test.
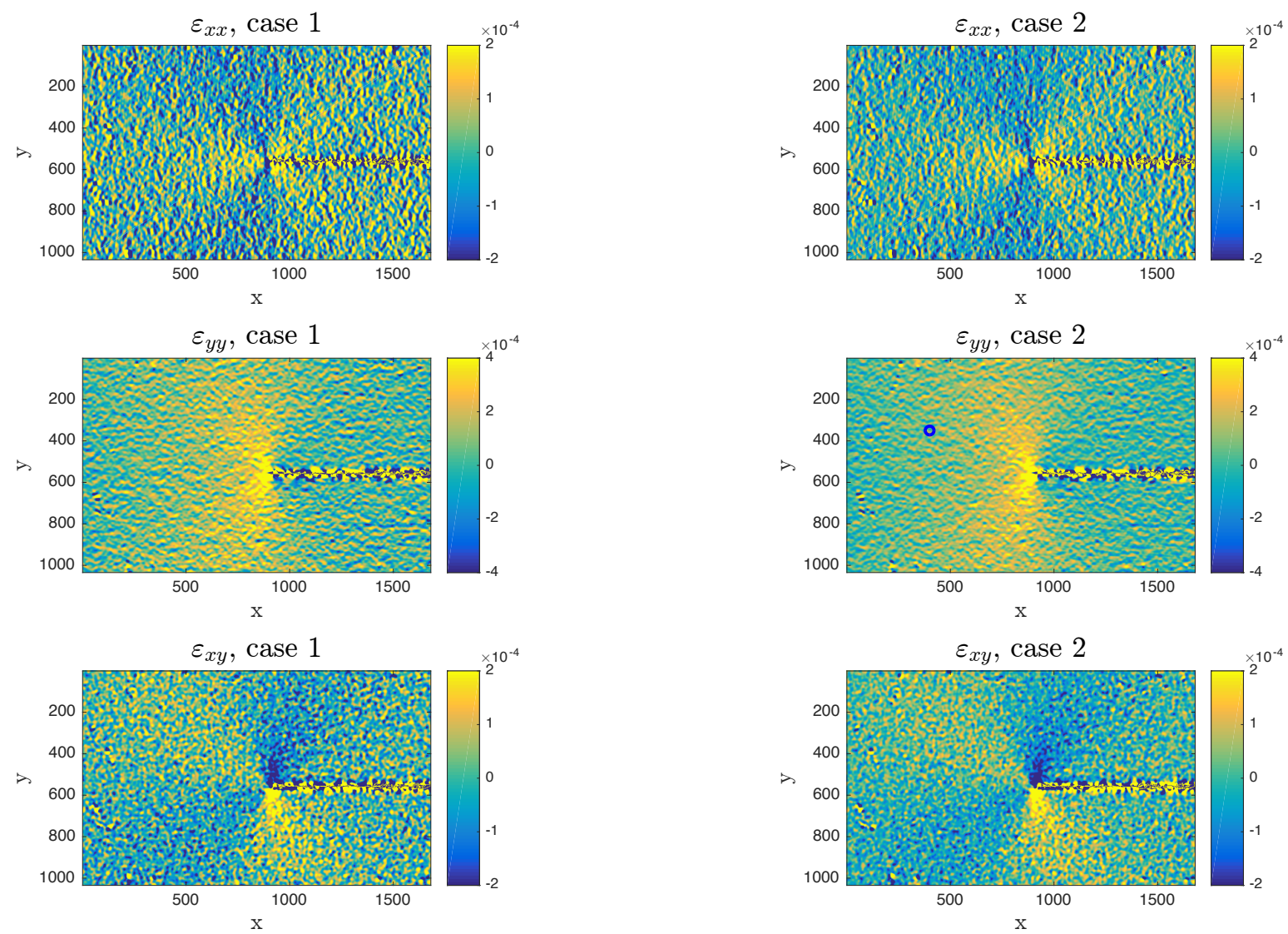

Figure 12: Typical strain maps obtained at the beginning of the tensile test. Case 1: without any time averaging. Case 2: with the initial phase distribution averaged with 100 successive phase maps. Standard deviation of the Gaussian window $\sigma=7$ pixels, pitch of the checkerboard: $p=9$ pixels. Blue circle: size of the Gaussian window used in the WFT according to the $3-\sigma$ rule [8]. All dimensions along $x$ and $y$ are in pixels $(1$ pixel $=22.2$ microns on the specimen).

The visual impact of the averaging procedure of the phase maps over 100 frames (case \#2) can be seen by comparing the corresponding strain maps with their counterparts obtained without averaging (case \#1). The defects in the pattern also propagate up to the final strain maps, "blobs" being observed at the location of these defects in the strain maps. Smaller "blobs" corrupt the three strain maps (the reader is invited to zoom in on the strain maps on the pdf file to observe them). They are due to sensor noise propagation, which manifests itself 
as a spatially correlated noise in the strain maps. These small "blobs" are elongated along the $y$-direction for $\varepsilon_{x x}$, elongated along the $x$-direction for $\varepsilon_{y y}$, but globally isotropic for $\varepsilon_{x y}$. These properties are justified in [11] for 2D grids but the explanations remain the same here for checkerboards. The diameter of the blue circle is $6 \times \sigma$. It represents the conventional width of the Gaussian envelope used in LSA according to the classic $3-\sigma$ rule [8], and thus the size of the optical displacement and strain gages available at any pixel. The main point here is that strain of small amplitude (some hundreds of microstrains) are measured at any pixel near the crack tip and over very small zone represented by the blue circle, which is $1.2 \mathrm{~mm}$ in size.

\section{Conclusion}

In this paper, it is shown that checkerboard images can be used to measure displacement and strain fields on flat deformed surfaces. A checkerboard is however a periodic marking, and the images were therefore not processed by DIC, but by using a Fourier-based method, namely the Localized Spectrum Analysis (LSA). Two procedures based on LSA were proposed, but the one which was finally used consists of applying LSA along the bisectors of the principal directions of symmetry of the checkerboard. Experiments show that displacement and strain maps obtained with a checkerboard are less noisy than their counterparts obtained with classic 2D grids. Checkerboards should therefore be used preferentially in place of $2 \mathrm{D}$ grids for displacement and strain measurement. Finally, strain maps obtained with a tensile test performed on a notched specimen show that such a pattern can be used in practice to measure strain distributions of low amplitude, but with high gradients. The proposed method is restricted to $2 \mathrm{D}$ measurements, so its extension to out-of plane measurements should also be investigated.

\section{References}

[1] G.F. Bomarito, J.D. Hochhalter, T.J. Ruggles, and A.H. Cannon. Increasing accuracy and precision of digital image correlation through pattern optimization. Optics and Lasers in Engineering, 91:73 - 85, 2017.

[2] B. Pan, H. Xie, Z. Wang, K. Qian, Z. Wang Study on subset size selection in digital image correlation for speckle patterns. Optal Express 16(10):703-7048, 2008

[3] M. Sutton, J.J. Orteu, H. Schreier Image correlation for shape, motion and deformation measurements. Basic concepts, theory and applications. Springer, 2009.

[4] Y.Q. Wang, M. Sutton, H. Bruck, H.W. Schreier Quantitative error assessment in pattern matching: effects of intensity pattern noise, interpolation, strain and image contrast on motion measurements. Strain 45(2):16-178, 2009

[5] J. Rï $₫ \frac{1}{2}$ thorï $\measuredangle \frac{1}{2}$. A fully integrated noise robust strategy for the identification of constitutive laws from digital images. International Journal for Numerical Methods in Engineering 84(6):63-660, 2010 
[6] M. Grédiac, B. Blaysat, and F. Sur. A critical comparison of some metrological parameters characterizing local digital image correlation and grid method. Experimental Mechanics, 57(3):871-903, 2017.

[7] F. Sur and M. Grédiac. Influence of the analysis window on the metrological performance of the grid method. Journal of Mathematical Imaging and Vision, 56(3):472-498, 2016.

[8] E. W. Grafarend. Linear and Nonlinear Models: Fixed Effects, Random Effects, and Mixed Models. Walter de Gruyter, 2006.

[9] Y. Surrel. Moiré and grid methods: a signal-processing approach. In Stupnicki J Pryputniewicz RJ, editor, Interferometry'94: photomechanics, volume 2342. SPIE, 1994.

[10] Y. Surrel. Design of phase-detection algorithms insensitive to bias modulation. Applied Optics, 36(4):805-807, 1997.

[11] M. Grédiac, F. Sur, and B. Blaysat. The grid method for in-plane displacement and strain measurement: a review and analysis. Strain, 52(3):205-243, 2016.

[12] F. Sur and M. Grédiac. Towards deconvolution to enhance the grid method for inplane strain measurement. Inverse Problems and Imaging, 8(1):259-291, 2014. American Institute of Mathematical Sciences.

[13] J.L. Piro and M. Grédiac. Producing and transferring low-spatial-frequency grids for measuring displacement fields with moiré and grid methods. Experimental Techniques, 28(4):23-26, 2004.

[14] F. Sur, B. Blaysat, and M. Grédiac. Determining displacement and strain maps immune from aliasing effect with the grid method. Optics and Lasers in Engineering, 86:317-328, 2016.

[15] X. Dai, H. Xie, H. Wang, C. Li, Z. Liu, and L. Wu. The geometric phase analysis method based on the local high resolution discrete fourier transform for deformation measurement. Measurement Science and Technology, 25(2):025402, 2014.

[16] X. Dai, H. Xie, and H. Wang. Geometric phase analysis based on the windowed fourier transform for the deformation field measurement. Optics and Laser Technology, 58(6):119-127, 2014.

[17] S. Avril, E. Ferrier, A. Vautrin, P. Hamelin, and Y. Surrel. A full-field optical method for the experimental analysis of reinforced concrete beams repaired with composites. Composites Part A, 35(7-8):873-884, 2004.

[18] F. Pierron, H. Zhu, and C. Siviour. Beyond Hopkinson's bar. Philosophical Transactions of the Royal Society A: Mathematical, Physical and Engineering Sciences, 372(2023):20130195, 2014.

[19] M. Rossi, F. Pierron, and P. Forquin. Assessment of the metrological performance of an in situ storage image sensor ultra-high speed camera for full-field deformation measurements. Measurement Science and Technology, 25(2):025401, 2014. 
[20] G. Le Louedec, F. Pierron, M.A. Sutton, C. Siviour, and A.P. Reynolds. Identification of the dynamic properties of Al 5456 FSW welds using the virtual fields method. Journal of Dynamic Behavior of Materials, 2015. doi: 10.1007/s40870-015-0014-6.

[21] B. Lukic, D. Saletti, and P. Forquin Use of simulated experiments for material characterization of brittle materials subjected to high strain rate dynamic tension Philosophical Transactions of the Royal Society A. Mathematical, Physical and Engineering Sciences. Jan 28;375(2085). pii: 20160168, 2017.

[22] H. Haddadi and S. Belhabib. Use of a rigid-body motion for the investigation and estimation of the measurement errors related to digital image correlation technique. Optics and Lasers in Engineering, 46(2):185-96, 2008.

[23] P. Reu. All about speckles: Aliasing. Experimental Techniques, 38(5):1-3, 2014.

[24] G.E. Healey and R. Kondepudy. Radiometric ccd camera calibration and noise estimation. IEEE Transactions on Pattern Analysis and Machine Intelligence, 16(3):267-276, 1994.

[25] J. Boulanger, C. Kervrann, P. Bouthemy, P. Elbau, J.-B. Sibarita and J. Salamero Patchbased nonlocal functional for denoising fluorescence microscopy image sequences. IEEE Transactions on Medical Imaging, 29(2), 442- 454.

[26] A. Foi, M. Trimeche, V. Katkovnik and K. Egiazarian. Practical Poissonian-Gaussian noise modeling and fitting for single-image raw-data. IEEE Transactions on Image Processing, 17(10), 1737-1754, 2008.

[27] S. Ramani, C. Vonesch, and M. Unser. Deconvolution of 3D fluorescence micrographs with automatic risk minimization. In Proceedings of the IEEE International Symposium on Biomedical Imaging (ISBI), 732-735, 2008.

[28] F. Sur and M. Grédiac. On noise reduction in strain maps obtained with the grid method by averaging images affected by vibrations. Optics and Lasers in Engineering, 66:210$222,2015$. 